\title{
Influence of butylated hydroxytoluene (BHT) on the viability of ram spermatozoa undergoing cold shock
}

\author{
P. F. Watson and W. J. Anderson
}

Department of Physiology, The Royal Veterinary College, Royal College Street, London NWI OTU, U.K.

\begin{abstract}
Summary. Butylated hydroxytoluene (BHT) significantly reduced the degree of acrosome damage which occurred to ram spermatozoa during cold shock. A higher percentage of spermatozoa were motile after cold shock in the presence of BHT than in its absence, but it had little effect on the quality of motility or the percentage of cells which stained with eosin. A concentration of 2-4 mM-BHT provided the maximum response. No advantage was gained by using the solvent, dimethyl sulphoxide, as a vehicle to introduce BHT to the cells. An osmotic stress test after cold shock also failed to demonstrate any advantage of BHT. It is concluded that BHT provides little protection to the functional capacity of ram spermatozoa undergoing cold stress and is unlikely to be of benefit for the preservation of ram semen.
\end{abstract}

\section{Introduction}

The sudden cooling of semen in the temperature range $20-0^{\circ} \mathrm{C}$, commonly referred to as cold shock, results in an irreversible loss of sperm motility, and the effects are particularly severe with spermatozoa of ungulate species, including the ram and bull. Therefore, considerable care must be exercised during semen preservation to avoid damaging the spermatozoa unduly at this stage of the processing. Although the mechanism is not entirely understood it is apparent that the cell membrane is fundamentally altered and the balance of evidence suggests that this has some relationship to lipid components and phase changes within the membrane (for review, see Watson, 1981).

Hammerstedt et al. $(1976,1978)$ proposed that the anti-oxidant, butylated hydroxytoluene (BHT), acted as a membrane lipid perturbant which would prevent (or substantially reduce) the permeability changes of bull or ram sperm plasma membranes when the cells were cold-shocked. Using an electron spin resonance technique they measured the proportion of the cell excluding $\mathrm{Ni}^{2+}$ or $\mathrm{Fe}(\mathrm{Cn})_{6}{ }^{3-}$ ions, and found that, before cold shock, the ions were excluded from the cells. After cold shock the ions gained free access to all aqueous compartments, indicating a profound change in membrane characteristics. The addition of $0.5 \mathrm{~mm}$-BHT to the diluent substantially prevented the changes occurring. However, BHT had no measurable effect on cold-shock damage in boar spermatozoa.

From these observations, Hammerstedt et al. (1978) suggested that BHT might ameliorate membrane damage during ram or bull semen processing, but was unlikely to be of value with boar semen. Pursel (1979) subsequently found that boar spermatozoa were protected by BHT, as judged by motility and acrosomal integrity, and the fertility of such cells was retained. In the light of these observations, we have also looked at a range of laboratory assessments of functional capacity of ram spermatozoa and the results are presented below. 


\section{Materials and Methods}

A simple diluent was used for all experiments, consisting of $325 \mathrm{~mm}$ Tris (Tris(hydroxymethyl)methylamine) titrated against $325 \mathrm{~mm}-\mathrm{HCl}$ to $\mathrm{pH} 7 \cdot 0$. The method of Hammerstedt et al. (1976) was used to induce the uptake of butylated hydroxytoluene (Sigma London Chemical Co., Poole, Dorset, U.K.) by spermatozoa. Briefly, $0.1 \mathrm{ml}$ volumes of ethanol (AR grade, BDH Chemicals Ltd, Poole, Dorset, U.K.) containing appropriate amounts of BHT were dried down under air in tubes before the addition of diluted semen. The amount of BHT present was specified by the particular experimental design and was sufficient to give the final concentration (0-8 $\mathrm{mm})$ in $1 \mathrm{ml}$ diluted semen. In Exp. 3, BHT was also dissolved in dimethyl sulphoxide (DMSO: BDH Chemicals Ltd),

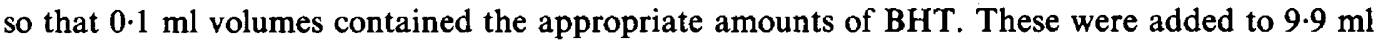
Tris- $\mathrm{HCl}$, the molarity of which had been reduced to accommodate the contribution that DMSO made to the final $325 \mathrm{~mm}$ diluent. (All molarities of BHT referred to are nominal owing to its marked insolubility in aqueous media.)

Semen was collected from Finnish Landrace rams by means of an artificial vagina. Only samples showing vigorous swirling motion were used. For Exps 1-3 semen was diluted 20-fold at $30^{\circ} \mathrm{C}$ either in Tris- $\mathrm{HCl}$ and added to BHT-coated tubes or in DMSO-Tris- $\mathrm{HCl}$ containing BHT. Duplicate $1-\mathrm{ml}$ volumes were placed in $10 \times 75 \mathrm{~mm}$ round-bottomed glass tubes, agitated for $30 \mathrm{sec}$ and held for $10 \mathrm{~min}$ at $30^{\circ} \mathrm{C}$.

Cold shock was generally achieved by immersion of the tubes in ice-water at $0^{\circ} \mathrm{C}$ for $5 \mathrm{~min}$. Other temperatures for cold shock were maintained within $\pm 1^{\circ} \mathrm{C}$ by adding ice to the water bath. After cold shock the tubes were warmed rapidly to $37^{\circ} \mathrm{C}$ and estimates of motility (score $0-4$ : Emmens, 1947) and percentage motile (to the nearest $10 \%$ ) were made from each of the tubes in random sequence. This procedure was repeated 3 times and the mean values were used for analysis. Eosin-nigrosin smears were prepared (Hancock, 1957) and counts were made in duplicate of the percentage of unstained cells. Samples were also fixed and stained with Giemsa for assessment of acrosomal deterioration (Watson, 1975). Individual cells were allocated a score of 0-3 according to the degree of damage. The results are expressed as the mean score of 20 cells chosen at random on the slide.

Experiments 4 and 5 (spermatocrit or packed cell volume determinations) were performed as follows. A sperm count was made in duplicate with a haemocytometer and the semen was diluted to a density of $200 \times 10^{6}$ spermatozoa $/ \mathrm{ml}$. After cold shock, NaF was added in solution (to give a final concentration of $20 \mathrm{mM}$ ) to arrest motility (Drevius, 1972), and the tubes were centrifuged on a bench centrifuge (MSE Minor) at approximately $800 \mathrm{~g}$ for $5 \mathrm{~min}$ (Exp. 4) or $10 \mathrm{~min}$ (Exp. 5). The supernatant was removed and $0.85 \mathrm{ml}$ Tris- $\mathrm{HCl}-\mathrm{NaF}$ (Tris- $\mathrm{HCl}$ at 325 or $65 \mathrm{~mm}$ according to the experimental design, together with $20 \mathrm{~mm}-\mathrm{NaF}$ ) was added at room temperature. The sperm pellets were readily dispersed and after $10 \mathrm{~min}$ the samples were recentrifuged for $5 \mathrm{~min}$. Exactly $0.8 \mathrm{ml}$ supernatant was removed using a Finnpipette (Buckley Scientific Ltd, Great Missenden, Bucks, U.K.) and the sperm pellet resuspended in the remaining diluent. Two Benjamin microhaematocrit tubes were filled from each sample and sealed with Plasticine. The haematocrit tubes were centrifuged in the bench centrifuge at $1500 \mathrm{~g}$ for $5 \mathrm{~min}$, held in specially constructed inserts for standard $15-\mathrm{ml}$ centrifuge tubes. Spermatocrit values were determined using a travelling microscope.

All data were analysed on a computer by analysis of variance. Percentage data were transformed to the arcsin $\sqrt{ } \%$ before analysis. The terminology, $L$ and $Q$, taken from Cochran \& Cox (1957), denotes the linear regression (L) and the deviation from linearity or quadratic curvature (Q). Because of the errors inherent in the processing of the semen before spermatocrit determinations in Exps 4 and 5, all treatments were carried out in triplicate for ejaculate. An hierarchical analysis of variance (Snedecor \& Cochran, 1976) was conducted to distinguish components of variance due to differences between treatments from those due to variations in the processing stages. 


\section{Results}

In Exp. 1 the influence of BHT on the effects of sudden cooling from 30 to $5^{\circ} \mathrm{C}$ was examined. BHT concentrations from 0 to $4 \mathrm{~mm}$ were used and the results are shown in Table 1. A significant linear response to BHT was seen in the percentage motile score $(P<0.01)$ and the acrosome score $(P<$ 0.05 ), indicating a beneficial effect of the inclusion of BHT. The motility score, however, showed an erratic response with no clear advantage with the inclusion of BHT. The percentage of unstained cells also failed to demonstrate any advantage of BHT and, if anything, suggested that BHT at $4 \mathrm{mM}$ was detrimental.

In Exp. 2 a concentration of 2 mM-BHT was chosen because this had given a clear response in Exp. 1. The semen samples were subjected to cold shock from $30^{\circ} \mathrm{C}$ to 0,5 or $10^{\circ} \mathrm{C}$. The rationale was to investigate the possibility of a differential response to the presence of BHT as the temperature of cold shock was reduced. The results are presented in Table 2. As the cold shock became more severe, so there was evidence of progressively more damage to the cells as shown by all scores. Once again BHT proved of significant advantage only for the percentage motile $(P<$

Table 1. Scores of structure and viability of ram spermatozoa after sudden cooling from 30 to $5^{\circ} \mathrm{C}$ in the presence of BHT (means of 2 ejaculates from each of 3 rams)

\begin{tabular}{|c|c|c|c|c|}
\hline & Motility $(0-4)$ & $\%$ motile & $\%$ unstained & Acrosome score $(0-3)$ \\
\hline After dilution before cooling & $3 \cdot 39$ & $72 \cdot 2$ & $72 \cdot 3$ & $1 \cdot 11$ \\
\hline \multicolumn{5}{|l|}{ BHT conc. (mM) } \\
\hline 0 & 1.96 & $25 \cdot 0$ & $6 \cdot 3$ & $1 \cdot 72$ \\
\hline 1 & $2 \cdot 28$ & $37 \cdot 2$ & $10 \cdot 1$ & 1.60 \\
\hline 2 & 1.97 & 38.6 & $10 \cdot 4$ & 1.36 \\
\hline 4 & $2 \cdot 01$ & $46 \cdot 1$ & $3 \cdot 0$ & $1 \cdot 29$ \\
\hline \multicolumn{5}{|c|}{ Significant Treatment contrasts from analyses of variance: } \\
\hline BHT vs none & NS & *** & NS & ** \\
\hline BHT conc. (L) & ** & ** & ** & * \\
\hline (Q) & * & NS & NS & NS \\
\hline
\end{tabular}

NS, Not significant; ${ }^{*} P<0.05,{ }^{* *} P<0.01,{ }^{* * *} P<0.001$.

Table 2. The effect of BHT on the structure and viability of ram spermatozoa cooled suddenly to 0,5 or $10^{\circ} \mathrm{C}$ (mean scores of two ejaculates from each of $3 \mathrm{rams}$ )

\begin{tabular}{ccccc}
\hline & Motility (0-4) & $\%$ motile & $\%$ unstained & Acrosome score (0-3) \\
\hline After dilution before cooling & 3.42 & 75.6 & 82.4 & 1.05 \\
\hline
\end{tabular}

BHT conc. (mM)

\begin{tabular}{|c|c|c|c|c|c|c|c|c|}
\hline $\begin{array}{l}\text { Final cooling } \\
\text { temp. }\left({ }^{\circ} \mathrm{C}\right)\end{array}$ & 0 & 2 & 0 & 2 & \multicolumn{2}{|l|}{$\mathbf{0}$} & 0 & 2 \\
\hline 0 & 1.72 & 1.69 & $21 \cdot 1$ & $24 \cdot 2$ & $7 \cdot 2$ & $8 \cdot 2$ & 1.61 & $1 \cdot 36$ \\
\hline 5 & 1.86 & 1.99 & $25 \cdot 3$ & $31 \cdot 1$ & $11 \cdot 2$ & 13.9 & 1.48 & $1 \cdot 34$ \\
\hline 10 & $2 \cdot 29$ & $2 \cdot 20$ & $35 \cdot 0$ & $41 \cdot 7$ & $18 \cdot 4$ & $25 \cdot 1$ & $1 \cdot 32$ & $1 \cdot 23$ \\
\hline \multicolumn{9}{|c|}{ Significant Treatment contrasts from the analyses of variance: } \\
\hline BHT vs none & \multirow{2}{*}{\multicolumn{2}{|c|}{ NS }} & \multirow{2}{*}{\multicolumn{2}{|c|}{$* *$}} & \multirow{2}{*}{\multicolumn{2}{|c|}{$\underset{* * *}{N S}$}} & \multicolumn{2}{|c|}{$* *$} \\
\hline Temperature (L) & & & & & & & \multirow{2}{*}{\multicolumn{2}{|c|}{ NS }} \\
\hline (Q) & \multicolumn{2}{|c|}{ NS } & \multicolumn{2}{|c|}{ NS } & \multicolumn{2}{|c|}{ NS } & & \\
\hline BHT $\times$ temp. interaction & \multicolumn{2}{|c|}{ NS } & \multicolumn{2}{|c|}{ NS } & \multicolumn{2}{|c|}{ NS } & \multicolumn{2}{|c|}{ NS } \\
\hline
\end{tabular}

NS, Not significant; ${ }^{*} P<0.05,{ }^{* *} P<0.01,{ }^{* * *} P<0.001$. 
$0.05)$ and acrosome $(P<0.01)$ scores. There was no evidence of any interaction between the temperature of cold shock and the presence of BHT.

Since the response to BHT was not obvious in all scores the possibility that insufficient BHT was gaining access to the cell membranes was considered in Exp. 3. As an alternative method of exposing spermatozoa to $\mathrm{BHT}$, the $\mathrm{Tris}-\mathrm{HCl}$ diluent was modified to incorporate a small proportion of DMSO in which BHT had previously been dissolved. This method was compared with the standard method, employing BHT concentrations ranging from 0 to $8 \mathrm{mM}$, on semen samples subjected to cold shock at $0^{\circ} \mathrm{C}$. The results are presented in Table 3 . There were no significant differences between the ethanol and DMSO methods of applying BHT in the scores relating to the quantity and quality of motility. The percentage of unstained cells was considerably greater with the DMSO treatment $(P<0.001)$ compared with the ethanol treatment but the degree of acrosomal disruption was significantly more pronounced $(P<0.001)$. The main effect of BHT was not significant except in the acrosome score for which BHT once again caused a marked reduction in the degree of cold shock damage $(P<0.001)$. Increasing the BHT concentration to 8 $\mathrm{mM}$ had no further beneficial effect beyond that seen with $2 \mathrm{mM}$, perhaps because of failure to dissolve increasing amounts of BHT. The interaction term between method of application of BHT and concentration was just significant $(P<0.05)$ for all scores except percentage motile, indicating a different pattern of responses to the concentrations of BHT between the methods of application. Significant differences were evident within and between individuals in Exps 1-3.

A further test of membrane function was devised in which spermatozoa which had been subjected to cold shock in the presence or absence of BHT were exposed to hypotonic media. The spermatocrit was then determined as a measure of the integrity of the sperm plasma membranes to act as semipermeable membranes and enable the spermatozoa to swell in response to the hypotonic media.

Experiment 4 was designed to investigate the effect on spermatocrit of exposure of the cells to media $20 \%$ of the molarity of the control diluent in the presence or absence of BHT. The results (Table 4) showed that BHT had no observable effect at room temperature. The spermatozoa responded to the hypotonic conditions by swelling which was clearly measurable by the spermato-

Table 3. The effect of method of application of BHT on the structure and viability of ram spermatozoa cooled suddenly to $0^{\circ} \mathrm{C}$ (mean scores of two ejaculates from each of 3 rams)

\begin{tabular}{|c|c|c|c|c|c|}
\hline $\begin{array}{l}\text { Method of } \\
\text { application } \\
\text { of BHT }\end{array}$ & $\begin{array}{l}\text { BHT conc. } \\
\text { (mM) }\end{array}$ & $\begin{array}{c}\text { Motility } \\
(0-4)\end{array}$ & $\%$ motile & $\%$ unstained & $\begin{array}{c}\text { Acrosome } \\
\text { score } \\
(0-3)\end{array}$ \\
\hline \multirow[t]{4}{*}{ Ethanol $\dagger$} & 0 & $1 \cdot 10$ & 21.9 & $8 \cdot 1$ & 1.59 \\
\hline & 2 & $1 \cdot 14$ & $26 \cdot 7$ & $8 \cdot 6$ & $1 \cdot 33$ \\
\hline & 4 & 0.81 & $22 \cdot 5$ & $10 \cdot 6$ & 1.42 \\
\hline & 8 & 0.85 & $23 \cdot 9$ & $7 \cdot 1$ & $1 \cdot 32$ \\
\hline \multirow[t]{4}{*}{ DMSO } & 0 & 0.90 & $22 \cdot 5$ & $10 \cdot 5$ & 1.60 \\
\hline & 2 & 0.89 & $23 \cdot 6$ & $14 \cdot 3$ & 1.52 \\
\hline & 4 & 0.89 & $22 \cdot 0$ & $10 \cdot 3$ & 1.56 \\
\hline & 8 & 0.97 & $26 \cdot 1$ & $16 \cdot 3$ & $1 \cdot 55$ \\
\hline \multicolumn{6}{|c|}{ Significant Treatment contrasts from the analyses of variance: } \\
\hline Method of application & & NS & NS & $* * *$ & $* * *$ \\
\hline BHT is none & & NS & NS & NS & $* * *$ \\
\hline BHT conc. (L) & & NS & NS & NS & NS \\
\hline$(\mathrm{Q})$ & & NS & NS & NS & NS \\
\hline \multicolumn{6}{|l|}{ Method of application } \\
\hline$\times$ BHT conc interaction & & * & NS & * & * \\
\hline
\end{tabular}

$\uparrow$ BHT dried down from ethanol into semen tubes before addition of diluted semen. $\ddagger$ BHT dissolved in DMSO and added to diluent.

NS, Not significant; ${ }^{*} P<0.05,{ }^{* *} P<0.01,{ }^{* * *} P<0.001$. 
crit method $(P<0.001)$. There were significant differences between ejaculates. The method was quite stable and capable of measuring real treatment differences, as shown by the much greater treatment mean squares compared with the between-tube and between-replicate mean squares.

The spermatocrit method was then used in Exp. 5 to investigate the effect of BHT on coldshocked spermatozoa. The results, shown in Table 5, indicated that, compared to the untreated

Table 4. The effects of osmotic stress and BHT on the spermatocrit $(\%)$ of diluted ram semen (means of ejaculates from 3 rams)

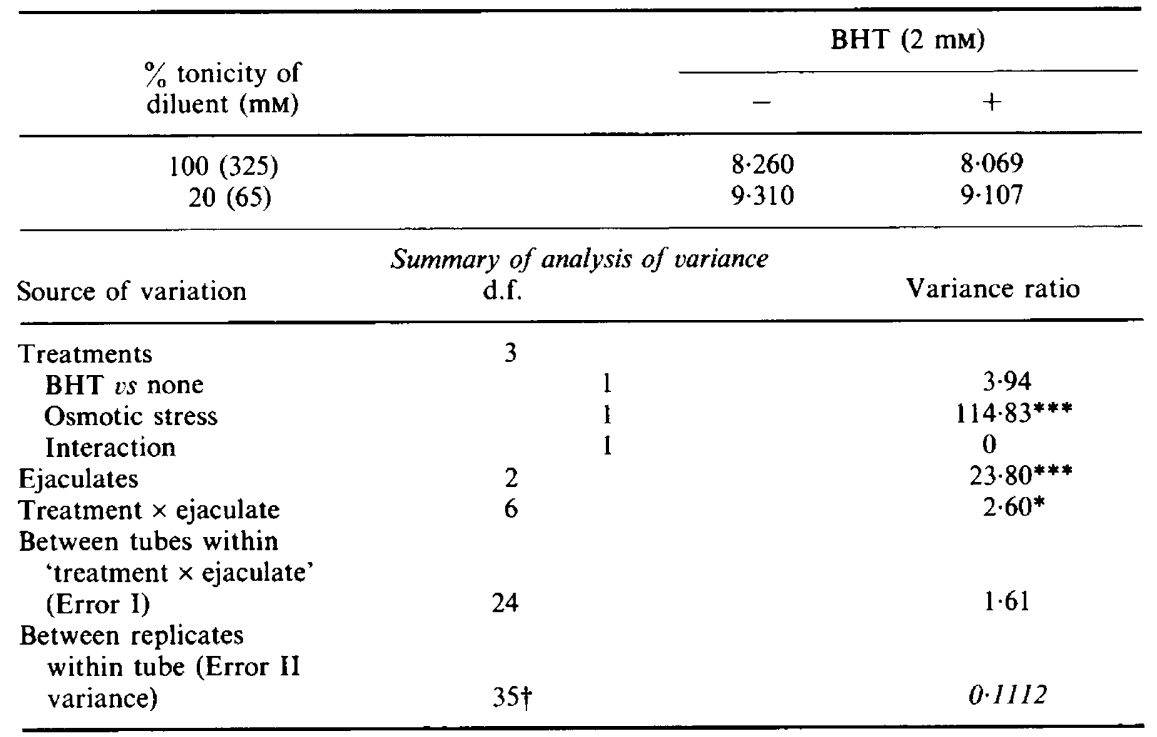

* $P<0.05,{ }^{* * *} P<0.001$.

$+36-1$ d.f. for a single estimated datum.

Table 5. Spermatocrit values (\%) of ram spermatozoa cooled suddenly in the presence of BHT and subsequently subjected to a hypotonic stress (means of ejaculates from 3 rams)

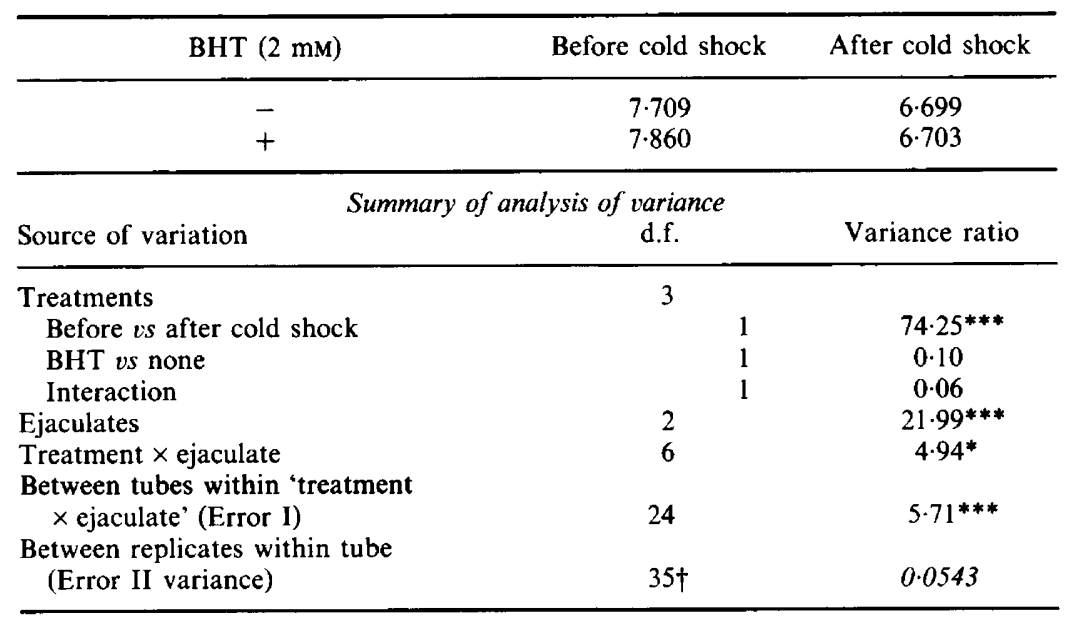

${ }^{*} P<0.05,{ }^{* * *} P<0.001$.

$\uparrow 36-1$ d.f. for a single estimated datum. 
controls, cold-shocked spermatozoa were unable to respond to the hypotonic conditions by swelling $(P<0.001)$ presumably because the membranes were damaged. BHT had no measurable influence on this lack of response.

\section{Discussion}

A uniform picture emerged in the results of Exps 1 and 2. Of the various aspects of structure and function determined, only the percentage motile score and the acrosome score showed a consistent beneficial effect of the presence of BHT. However, the scale of the response was relatively small even though significant differences were recorded. In contrast, the electron spin resonance results of Hammerstedt et al. $(1976,1978)$ indicated that the inclusion of $0.5 \mathrm{mM}$-BHT reduced the effect of cold shock in bull and ram spermatozoa by more than $80 \%$. Using $\left[{ }^{3} \mathrm{H}\right] \mathrm{BHT}$ these workers demonstrated that bull spermatozoa bound approximately $30 \%$ of the available BHT in these circumstances, and assumed that a similar uptake occurred in spermatozoa of other species. We did not consider it necessary to go to the considerable expense of confirming the uptake of BHT with a tritiated preparation since (1) the response in these and other experiments was consistently positive, and (2) our diluent, although differing from that of Hammerstedt et al. $(1976,1978)$, was a buffered salts solution of similar $\mathrm{pH}$, tonicity and ionic strength, factors which might influence the transference of BHT to the cells. Pursel (1979) also assumed the binding of BHT to boar spermatozoa although his diluents differed markedly from that of Hammerstedt et al. (1978).

However, the use of DMSO as a vehicle to introduce BHT was investigated to determine whether an improved uptake of BHT might increase the scale of the resistance to cold shock. DMSO was chosen as a solvent since it is widely used as a cryoprotectant, is no more toxic for ram spermatozoa than glycerol and freely permeates the cell membranes (Jones, 1965). As the results of Exp. 3 indicated, the use of DMSO to introduce BHT did not increase the resistance of cell membranes to cold shock beyond that achieved with the original method. The significant interactions in this experiment are not easily explained. The presence of DMSO appeared to prevent the decline in the quality of motility with increasing BHT concentration but there was no similar response in the percentage of motile spermatozoa. The effects of DMSO on the percentage of unstained cells may or may not reflect a real effect on the maintenance of cell membrane function. This result could be explained by the interference of DMSO with the staining process and, in the light of other experiments not reported here, this is the view favoured by the authors. The consistently poorer acrosomal condition also suggests that the membranes were not protected from the effects of cold shock by the presence of DMSO.

Spermatocrit determinations have been shown accurately to reflect changes in cell volume, providing that (1) the medium is not so hypotonic that it induces cell lysis and (2) the centrifugal forces employed are moderate (Drevius, 1972). The method used here was considered to fulfil these conditions, and although the results were not corrected for trapped extracellular medium they provided a ready means of comparing cell volumes of spermatozoa subjected to varying tonicity of the medium (Table 4). The spermatocrit measurement was used to test the ability of the plasma membrane to act as a semipermeable membrane. This function depends on the integrity of the plasma membrane which is destroyed when ram spermatozoa are cold-shocked. It was shown in Exp. 5 that BHT was not able to prevent this loss of membrane integrity.

It is clear from these results that BHT introduced a minor but significant resistance to membrane disruption during cold shock. Hammerstedt et al. (1976) recorded decreased viscosity of sperm membrane lipids in the presence of BHT. They speculated that this may imply greater lipid fluidity at reduced temperature and, therefore, greater resistance to the development of membrane pores or ruptures. However, the membrane disruption which occurs to ram spermatozoa during cold shock is extensive, and is difficult to account for by concepts of lipid-phase changes alone (Watson, 1981). The metabolic studies of Hammerstedt et al. (1976) suggested that in the presence 
of BHT the membrane permeability changes were less marked but other metabolic effects of cold shock were unaffected. Our results also bear this out. The gross structural changes were less pronounced, i.e. the overall degree of damage to the cell population was reduced, and this probably explains why more cells retained motility. Other assessments of cell and membrane function, e.g. quality of motility, eosin stainability, and response to osmotic stress, were unchanged, and indicated that in spite of apparently greater structural cohesion after cold shock it was not accompanied by a comparable retention of function.

It appears, therefore, that the use of electron spin resonance to determine $\mathrm{Ni}^{2+}$ or $\mathrm{Fe}(\mathrm{Cn})_{6}{ }^{3-}$ inaccessible space within spermatozoa is capable of registering gross permeability changes in sperm membranes but the results are not correlated with the subtle functional changes that occur after cold shock even in the presence of BHT. Our observations of membrane function lead us to conclude that BHT is unlikely to be of benefit to the preservation of ram spermatozoa. This conclusion must be tempered by knowledge of the observations made of boar spermatozoa (Pursel, 1979 ) in which both motility and acrosomal structure were retained after cold shock in the presence of BHT, and this was shown to be correlated with the retention of fertility. The degree of response to BHT was, however, considerably greater than we were able to record.

We thank Dr I. C. A. Martin, University of Sydney, for the computer programme used to analyse the results.

\section{References}

Cochran, W.G. \& Cox, G.M. (1957) Experimental Designs, 2nd edn. John Wiley \& Sons, New York.

Drevius, L.-O. (1972) Bull sperm as osmometers. $J$. Reprod. Fert. 28, 29-39.

Emmens, C.W. (1947) The motility and viability of rabbit spermatozoa at different hydrogen-ion concentrations. J. Physiol., Lond. 106, 471-481.

Hammerstedt, R.H., Amann, R.P., Rucinsky, T., Morse, D.P., Lepock, J., Snipes, W. \& Keith, A.D. (1976) Use of spin labels and electron spin resonance spectroscopy to characterise membranes of bovine sperm: effect of butylated hydroxytoluene and cold shock. Biol. Reprod. 14, 381-397.

Hammerstedt, R.H., Keith, A.D., Snipes, W., Amann, R.P., Arruda, D. \& Griel, L. (1978) Use of spin labels to evaluate effects of cold shock and osmolality on sperm. Biol. Reprod. 18, 686-696.

Hancock, J.L. (1957) The morphology of boar spermatozoa. Jl R. microse. Soc. 76, 84-97.
Jones, R.C. (1965) The use of dimethyl sulphoxide, glycerol, and reconstituted skim milk for the preservation of ram spermatozoa. I. The tonicity and toxicity of dimethyl sulphoxide and reconstituted skim milk at 30 and $5^{\circ}$ C. Aust. J. biol. Sci. 18, 877885.

Pursel, V.G. (1979) Effect of cold shock on boar sperm treated with butylated hydroxytoluene. Biol. Reprod. 21, 319-324.

Snedecor, G.W. \& Cochran, W.G. (1967) Statistical Methods, 6th edn. Iowa State University Press, Ames.

Watson, P.F. (1975) Use of a Giemsa stain to detect changes in the acrosomes of frozen ram spermatozoa. Vet. Rec. 97, 12-15.

Watson, P.F. (1981) The effects of cold shock on sperm cell membranes. In Effects of Low Temperatures on Biological Membranes, pp. 189-218. Eds G. J. Morris \& A. Clarke. Academic Press, London.

Received 4 February 1983 